\title{
Equidad y movilidad intergeneracional de los titulados universitarios catalanes ${ }^{1}$
}

\author{
Sandra Fachelli \\ Jordi Planas \\ Universitat Autònoma de Barcelona. Grup de Recerca sobre Educació i Treball (GRET) \\ jordi.planas@uab.cat; sandra.fachelli@uab.cat
}

\section{Resumen}

Este artículo aborda la igualdad de oportunidades y la movilidad profesional de los universitarios catalanes en el acceso a su graduación y su inserción profesional en comparación con la de sus padres. El análisis se realiza en base a una encuesta realizada por la AQU del Gobierno catalán sobre la situación en 2008 de los graduados en 2004.

Palabras clave: titulados universitarios; diferencias generacionales; mercado de trabajo; movilidad laboral; Cataluña.

\section{Abstract. Intergenerational Equity and Mobility of the Catalan Graduates}

This article addresses the equal opportunity and professional mobility of Catalan university students by comparing their graduation rates and employability to those of their parents. This analysis is based on a survey carried out by AQU of the Catalan Government in 2008, pertaining to the situation of graduates in 2004 .

Keywords: universities graduates; generational differences: labour market; occupational mobility; Catalonia.

\author{
Sumario \\ 1. Introducción 4. Resultados \\ 2. Educación, equidad y movilidad \\ 5. Conclusiones \\ intergeneracional \\ Referencias bibliográficas \\ 3. Metodología \\ Anexo 1. Ficha técnica del estudio
}

1. Este artículo ha sido realizado en base a los microdatos de la encuesta llevada a cabo por la Agencia para la Calidad del Sistema Universitario de Cataluña (AQU) del Gobierno catalán y su apoyo financiero, y también en el marco del proyecto Itinerarios universitarios, Equidad y Movilidad Ocupacional (ITUNEQMO) CSO2010-19271, financiado por Plan $\mathrm{I}+\mathrm{D}+\mathrm{I}$ del Ministerio de Ciencia e Innovación. 


\section{Introducción}

La transición del sistema educativo al trabajo, o transición laboral de los jóvenes, constituye uno de los fenómenos más determinantes en la construcción de la vida adulta de las personas y, consecuentemente, del futuro de nuestras sociedades. Su estudio, por lo tanto, se inscribe en el interés por parte de los científicos sociales por describir e interpretar el proceso a través del cual los jóvenes dejan de ser jóvenes, interés que se refleja en la abundante literatura sobre el tema, desde el estudio pionero de Coleman (1979), hasta los trabajos realizados por Raffe (2003, 2011). Esta transición constituye un punto de observación privilegiado para los análisis de las funciones desempeñadas por tres instituciones fundamentales de nuestras sociedades: la familia, el ámbito educativo y el mercado de trabajo.

La manera concreta con la cual un individuo desembarca en el periodo adulto de su vida es el resultado ciertamente de sus propias decisiones, así como de los contextos sociales y culturales que las inspiran (la clase social, el género, el lugar de origen, etc.). Pero también depende de las configuraciones institucionales que limitan y canalizan estas mismas decisiones: las oportunidades educativas, las estrategias de los empleadores que los deben contratar o no, la estructura y las preferencias de la población activa que compite con él, las políticas públicas de apoyo a la ocupación juvenil, etc.

En definitiva, la transición de la escuela $-\mathrm{y}$ de la universidad en particular - al mundo del trabajo constituye un objeto de estudio que implica dimensiones diversas, que se presta a perspectivas a menudo contrapuestas y que remite a la complejidad y a la variabilidad de nuestros mercados laborales y de nuestras sociedades en su conjunto.

Para centrar un objeto de estudio tan complejo como es la transición laboral de los jóvenes, resulta imprescindible situarlo en una perspectiva histórica (Sala et al., 2007).

Situándola históricamente, la generación de la cual analizamos su inserción profesional, con respecto a sus estudios, es una generación que se ha educado en pleno auge de la escuela de masas, bien al contrario de lo que les sucedió a sus padres, que nacieron a finales de los años cuarenta y accedieron a la escuela durante los años cincuenta y sesenta, caracterizados por la miseria educativa del franquismo.

La expansión educativa constituye uno de los fenómenos sociales más decisivos de la segunda mitad del siglo XX en las sociedades europeas. Es el resultado de un consenso histórico entre los estados, las organizaciones productivas y los individuos y las familias, todos ellos interesados en contribuir a aumentar los niveles académicos. Se trata, por lo tanto, de una extensión masiva de la formación, tanto entre la población (a través de la universalización del sistema educativo), como en el tiempo (la duración mediana de los estudios no ha parado de aumentar desde la década de 1960). En el caso español, se trata de un crecimiento del nivel educativo que se inicia más tarde 
que en los países de su entorno, pero que es más acelerado ${ }^{2}$, lo que provoca que las diferencias en las oportunidades académicas de las generaciones estudiadas y las de sus padres hayan sido muy superiores a las que podemos observar en países europeos para las mismas generaciones (Béduwé y Planas, 2003: 173-175).

Desde los años sesenta y, sobre todo, durante el período democrático postfranquista, se ha producido en Cataluña un gran crecimiento del número de alumnos a todos los niveles y, particularmente, el de la universidad, como resultado de una transformación cuantitativa basada en el crecimiento de la oferta pública de plazas de las universidades catalanas, tanto de cada universidad como del número de universidades. Pero también se ha transformado la función social de dichas instituciones, que han abandonado su carácter elitista. Durante el periodo democrático, el número de estudiantes universitarios se ha triplicado, tanto en España en general como en Cataluña en particular (Rotger, 2009; INE, 1976 y 2009; IDESCAT —consultado en línea en diciembre de 2010). Debido a esto, si analizamos la inserción de los titulados de la universidad actual, deberemos tener presente los efectos de los cambios en su origen social en esta inserción. Para entender la inserción profesional de los titulados de la vieja universidad elitista, debemos tener en cuenta, además de la calidad de sus estudios, la capacidad económica de sus familias (capital económico), sus relaciones sociales (capital social) y el nivel de estudios de sus padres (capital cultural). Comparativamente, las familias de los titulados de la nueva universidad de masas disponen de un capital económico, social y cultural comparativamente inferiores al de los titulados de la universidad elitista de la generación de sus padres. Este factor debería considerarse a la hora de comparar la inserción de los titulados universitarios de la generación de sus padres con la de los nuevos titulados, aun cuando, como muestran los resultados que se presentan acto seguido, es bastante buena.

Este fenómeno es compartido, al menos, por todos los países de la OCDE (2010), lo que da una mayor significación al caso particular que presentamos en este texto.

En particular, respecto al sistema universitario, los cambios institucionales que mayor impacto han tenido en el acceso a la enseñanza universitaria en España durante las últimas décadas han estado relacionados con: $a$ ) la estructura interna de los ciclos universitarios, $b$ ) la flexibilidad en la conexión entre ciclos universitarios y de éstos con los no universitarios, $c$ ) las vías y condiciones de acceso a la universidad, $d$ ) el crecimiento de la oferta universitaria y $e$ ) la proximidad geográfica de la oferta a sus usuarios potenciales, a través de su descentralización.

2. Aunque el nivel de estudios de los jóvenes españoles hoy es análogo, o incluso superior si nos referimos a la educación universitaria, a los países de la UE, la expansión educativa empezó en España con varias décadas de retraso respecto a dichos países. Debido a ello, las oportunidades para estudiar de los padres de los titulados universitarios fue menor que las de sus coetáneos europeos, considerados globalmente y teniendo en cuenta las distancias entre padres e hijos mayores. 
En estas páginas, analizaremos la inserción profesional en 2008 de la generación de los titulados universitarios en 2004 y la compararemos con la de sus padres; podemos considerarla una cuestión emblemática, porque, en este caso, la distancia entre la fecha de nacimiento de los padres y la de la graduación de los hijos abarca el medio siglo que ha sido dominado por la expansión educativa, los padres nacidos alrededor del 1950 y los hijos graduados en 2004.

Para las generaciones analizadas, el porcentaje de acceso a la universidad se sitúa alrededor del 40\% (Instituto Nacional de Estadística, 2005). La escolarización prolongada aumenta sustantivamente para las mujeres y los hijos de las clases populares y deviene poco menos que «deber social» de las clases medias y altas (con la intención de evitar la movilidad descendente). Este crecimiento se ha fundamentado en la certeza acerca del valor tanto social como individual de la educación. En nuestras sociedades, sobre todo en momentos de crisis, la incertidumbre sobre los cambios sociales y económicos que habremos de superar se acompaña de la confianza en que la elevación del nivel de educación del conjunto de la población, pero sobre todo de los jóvenes, es un factor fundamental para afrontarlos. Son ilustrativas de este planteamiento tanto las propuestas de la Estrategia 2020 de la Unión Europea, como las de la Ley de economía sostenible recientemente aprobada en España.

Aun así, a diferencia de la fase de la primera escuela de masas, este nuevo contexto origina una creciente complejidad en las relaciones entre la formación y la ocupación (Planas et al., 2001; Sala, 2011), que se reflejan en su alejamiento de las relaciones formalmente prescritas, como pueden ser los cuadros nacionales de cualificaciones (Sala et al., 2010), excepto para los empleos legalmente reglamentados. ¿En qué consiste la adecuación entre la formación recibida y el empleo ejercido? Este ha sido un tema recurrente en las ciencias sociales que ha suscitado grandes debates, tanto científicos como políticos, aunque no se han alcanzado resultados concluyentes (Hartog, 2000; Sala, 2011). Según los datos disponibles en investigaciones anteriores, durante las últimas décadas observamos que el proceso de ajuste entre formación y empleo se ha venido produciendo de manera bastante satisfactoria, al menos a medio plazo, en Europa y los Estados Unidos (Béduwé y Planas, 2003). Estudios recientes referentes al nivel universitario (Planas y Fachelli, 2010) y no universitario (Sala et al., 2010) nos indican que, al menos desde el punto de vista de los jóvenes empleados, el grado de adecuación entre el empleo ocupado y la formación recibida es elevado (en torno al $80 \%$ lo consideran adecuado en ambos casos), lo que no excluye una parte de inadecuaciones ni tensiones en dicha relación (Planas et al., 2001).

El período de inserción profesional que analizamos, entre el 2004 y el 2008, ha sido probablemente uno de los más favorables a la inserción profesional de los jóvenes titulados universitarios, por dos razones: por un lado, la caída de la natalidad empieza a reducir el número de titulados y, por otro lado, porque es un período de crecimiento económico; aunque dentro de un funcionamiento del mercado de trabajo, sobre todo para los jóvenes, marcado por la precariedad en la ocupación. Al mismo tiempo, es un período de crecimiento, tanto de la 
tasa de actividad como de la ocupación, sobre todo para las mujeres (Esteban et al., 2009).

Los datos que presentamos a continuación nos permiten tener una idea más clara sobre si nuestro sistema universitario (el conjunto de nuestras universidades) desempeña el papel de ascensor social que algunos le otorgan, o si, como mantienen otros, bien al contrario, reproduce las diferencias derivadas del origen social de los jóvenes, puesto que filtran su acceso y/o condicionan su rendimiento académico.

Este artículo aporta elementos de análisis, a partir del caso de las universidades catalanas, acerca del grado de "equidad» y de «empleabilidad" ${ }^{3}$ de los graduados formados antes de la implantación del proceso de Bolonia, lo que nos sirve para plantear una serie de interrogantes acerca de los posibles efectos de su aplicación.

\section{Educación, equidad y movilidad intergeneracional}

El papel de la escuela en nuestras sociedades ha sido uno de los temas centrales de las ciencias sociales, en general, y de la sociología, en particular. En este texto, abordamos dos de los dilemas que se han planteado las ciencias sociales acerca del sistema educativo y de la universidad: por un lado, el de la equidad en el acceso a la educación superior y, por otro, el de la movilidad ocupacional derivada de la educación universitaria.

Por lo que respecta a la equidad, la pregunta central que se ha planteado, desde los años setenta, respecto al sistema educativo en general y sobre la universidad en particular, ha sido: ¿La escuela es una institución social que ofrece las mismas oportunidades a todos los niños y jóvenes independientemente de su familia de origen, o, por el contrario, refuerza, y legitima, las desigualdades derivadas del origen social de los estudiantes?

Frente a esta pregunta, distintas corrientes teóricas, e ideológicas, han puesto el acento en cada una de las opciones.

Ya desde 1959, Parsons (1959), dentro de la corriente funcionalista y meritocrática, puso el énfasis en el papel de la escuela como distribuidor entre sus alumnos de las funciones requeridas por la división del trabajo en un marco meritocrático, es decir, que proporcionara a los estudiantes las mismas oportunidades de acceso y éxito independientemente de su familia de origen.

Posteriormente, a partir de los años setenta, mayormente dentro de la corriente marxista - aunque también desde el funcionalismo crítico (Coleman, 1973, 1982) —, se formularon las teorías de la reproducción (Bowles y Gintis, 1976; Baudelot y Establet, 1979), que criticaban a las teorías funcionalistas y ponían el acento en el carácter discriminante de la escuela como reproductora de las desigualdades de origen de los niños y los jóvenes, al ofrecerles distintas

3. En este texto, utilizamos el concepto de «empleabilidad» en el significado originario que le dieron Hillage y Pollard (1998), que se asocia a la capacidad personal para obtener un primer trabajo y para mantenerse empleado en el mismo o en otro. 
oportunidades de acceso y de resultados en función de su origen social. De esta manera, dichas investigaciones, y muchas más que han abundado posteriormente en esta corriente de pensamiento, nos muestran el sistema educativo como un sistema social que reproduce y legitima en los hijos las diferencias sociales de sus padres.

Por otra parte, la literatura sociológica ha estado tradicionalmente muy implicada en el análisis de la relación entre nivel educativo y estatus ocupacional y ha encontrado una relación muy elevada entre ambos elementos (Boudon, 1983: 40).

Investigaciones pioneras en este aspecto, como las de Blau y Duncan (1967), utilizando el método causal de análisis, observan la incidencia de cuatro variables sobre el estatus ocupacional de los hijos (educación y ocupación del padre, y educación y primer trabajo del hijo) y pueden explicar un $43 \%$ de la varianza total y, aunque esta técnica se mejora con el modelo del logro educativo de Wisconsin, en 1975, al agregar variables psicológicas, consiguen explicar el $40 \%$ del logro ocupacional y el $57 \%$ del logro educativo. Los autores observan que los efectos del estatus socioeconómico de los padres en el logro educativo y ocupacional del hijo operan a través de otras variables (referentes principales del hijo — sus pares-) que influyen sobre las aspiraciones educativas y ocupacionales de los hijos (Kerbo, 2003: 174-177). Por su parte, una revisión del modelo de Wisconsin analiza una generación anterior a la de los padres y se encuentra que el estatus socioeconómico de los abuelos no influye en el logro ocupacional y educativo de los nietos (Warren y Hauser, 1997: 561-572).

Resulta interesante recordar que, tradicionalmente, la literatura sociológica ha tomado la ocupación del jefe de familia como eje para estratificar a la sociedad o, en general, la de los trabajadores hombres. Una de las líneas de trabajo más prolífera en esta temática han sido los aportes John Goldthorpe y sus colegas del Nuffield College de Oxford. Este autor considera que la ocupación de una posición en el ámbito laboral es un indicador bastante apropiado para evaluar la posición social del individuo.

Goldthorpe desarrolla un esquema de clases, posteriormente mejorado, pero que inicialmente constaba de tres categorías, tomando al jefe de hogar varón como unidad de análisis: clase de servicios (con las clases I y II en su clasificación), clases intermedias (clases III a V) y clase trabajadora (clases VI y VII) (Goldthorpe, 1980: 39-42). Pero los grandes cambios acontecidos en la sociedad modifican la perspectiva sobre el tema y se toma conciencia que esta división del trabajo por género respondía a la separación de las esferas de actividad del hombre y de la mujer, la cual fue considerada como natural (Crompton, 1999: 105), de manera tal que, en décadas más recientes, empieza a incorporarse a la mujer en este tipo de análisis. Concretamente, en un trabajo relativamente reciente, Goldthorpe y Jackson analizan la movilidad social por género y encuentran que, contrariamente a lo que se asume habitualmente, no hay evidencia de que las tasas absolutas de movilidad estén cayendo; aunque el balance entre movimientos ascendentes y descendentes para los hombres se 
está volviendo menos favorable. Con respecto a las tasas de movilidad relativas, afirma que, tanto para los hombres como para las mujeres, permanecen esencialmente constantes, aunque es posible percibir indicios de una propensión al descenso de la movilidad a largo plazo (Goldthorpe y Jackson, 2007: 539-543).

Carabaña, reconociendo que el esquema de Erikson y Goldthorpe es el más utilizado en los análisis de movilidad, adapta las categorías de la encuesta sociodemográfica realizada por el INE en 1991 y llega a una división en tres subgrupos: clase de servicios, intermedia y trabajadora (Carabaña, 1999: 42-52). Compara el caso español con el resto de los países analizados por Erikson y Goldthorpe y, refiriéndose a la movilidad absoluta global, concluye que «[...] el inicio de la industrialización aumenta la movilidad ascendente, pero, cuanto más industrializado está un país, menor es su movilidad ascendente y mayor su movilidad descendente, de modo que la movilidad total tiende a descender» (Carabaña, 1999: 93).

De hecho, los estudios de movilidad empezaron a desarrollarse a partir de la Segunda Guerra Mundial y buscaban explicaciones sobre el desarrollo de las sociedades industriales. Algunos ejemplos de esta línea de trabajo son los estudios de David Glass y su equipo del London School of Economics en 1949, el trabajo de Lipset y Zetterberg en 1956 y el de Lipset y Bendix en 1959. Glass, por ejemplo, combinó categorías educativas y categorías socioprofesionales para realizar este tipo de estudios (Hernández de Frutos, 1997: 152-154).

En general, la ocupación, como hemos mencionado anteriormente, ha sido el indicador más utilizado para determinar la clase social y el primer elemento para determinar la posición del individuo en la estructura social. La profesión u ocupación es considerada un concepto más completo y preciso que los ingresos. De esta manera, la ocupación es algo más que una manera de ganar dinero, constituye un índice y un símbolo de la forma de vivir de la gente y el grado de prestigio que le asignan los otros (Hernández de Frutos, 1997: 153).

A pesar de la notoriedad de este tipo de análisis, el amplio uso de estos indicadores de ocupación ha sido aplicado solo al hombre, como se ha manifestado, y ello ha dado lugar a una «[...] identificación reduccionista entre movilidad social y movilidad masculina, que ha tenido como consecuencia más inmediata la práctica invisibilidad de la mitad femenina como objeto de estudio» (Salido Cortés, 2001: 43).

En general, las relaciones entre género, valores y estructura social han estado muy poco estudiadas (Xiao, 2000) y hasta ahora una minoría de estudios ha analizado la contribución relativa de ambos padres (Kalmijn, 1994), con lo cual ha dejado en el desconocimiento la influencia del estatus socioeconómico de las madres en los resultados sociales y psicológicos de sus hijos (Hitlin, 2006: 29).

Hay toda una corriente de análisis de la transición de la dependencia hacia la independencia de los jóvenes que pone especial atención en la formación de sus valores. Hitlin afirma que los valores y las aspiraciones, aunque socialmente formados y encauzados, representan las orientaciones emocionales y cogniti- 
vas del agente individual (el joven) que forma decisiones ocupacionales. Los jóvenes seleccionan carreras (o itinerarios), dadas sus habilidades y capacidades, cuando tienen las oportunidades estructurales para hacerlo y cuando las perciben como reales (Hitlin, 2006: 26). El autor, haciendo una exégesis de la relación entre el estatus socioeconómico y el desarrollo de los valores y las aspiraciones, recoge importantes contribuciones y afirma que muchos trabajos siguen el enfoque desarrollado por Melvin Kohn, que estudia la relación entre el estatus socioeconómico familiar y dos valores particulares: la conformidad y la autonomía. Una posición en la estructura de clases influencia la adopción de estos valores ofreciendo (o bloqueando) las oportunidades de experimentarlos dentro de una ocupación (Kohn, 1969, 1976, 1977, 1981; Kohn y Schooler, 1982, 1983; Kohn et al., 1990; Pearlin y Kohn, 1966; Slomczynski et al., 1981). Los valores de conformidad o de autonomía de los padres son formados por una sustantiva complejidad del contexto ocupacional y de clase y son transmitidos dentro de la familia (Kohn y Schoenbach, 1993; también ver Johnson 2002). Tendremos oportunidad de realizar este tipo de análisis a partir de una investigación incipiente que estamos llevando a cabo, donde el abordaje cualitativo nos permite indagar en los valores y las aspiraciones de los jóvenes al construir sus itinerarios vitales.

\section{Metodología}

En este trabajo, queremos recoger la tradición del análisis ocupacional y educativo de los jóvenes evitando los sesgos sexistas. Ahora bien, el análisis del estatus socioeconómico de una población determinada según la aplicación de uno u otro indicador (o ambos) aportan resultados diferentes. El indicador de educación referencia el origen familiar de los estudiantes a su edad temprana, en términos de capital cultural, y es relativo a las oportunidades educativas disponibles para cada generación. En cambio, el indicador de ocupación refleja una situación que puede modificarse con mayor facilidad que el nivel educativo, los padres pueden variar su puesto de trabajo durante toda su vida profesional, pero alcanzan un determinado nivel educativo durante su juventud y es difícil que lo modifiquen posteriormente.

La encuesta realizada a titulados universitarios, como hemos manifestado, dispone de las variables nivel educativo y ocupación de los padres, en consecuencia, nuestro trabajo aborda el origen familiar de los titulados a partir de analizar estas dos dimensiones. En el caso particular de nuestro análisis, la ocupación es un indicador del nivel socioeconómico de los padres en el momento de la encuesta (2008), mientras que el indicador del nivel educativo se refiere a la juventud de los padres. No obstante, existe una relación entre ambos indicadores ( $\mathrm{R}$ de Pearson: 0,51).

En términos metodológicos, hace falta destacar que la respuesta de los titulados universitarios sobre ambos temas (educación y ocupación de sus padres) supera el 98\%, con lo cual se ha podido establecer una buena base para realizar el análisis que presentamos a continuación. 
En nuestro análisis, la variable que nos permite identificar la educación de los padres de los graduados no diferencia entre padre y madre y está clasificada en cinco categorías: los dos padres tienen estudios primarios o no tienen estudios, uno de los dos tiene estudios medios, los dos tienen estudios medios, uno de los dos tienen estudios superiores y los dos tienen estudios superiores. Con el fin de lograr una mayor capacidad descriptiva y expositiva del origen de procedencia de los titulados universitarios en función del nivel educativo de sus padres, estas categorías se agrupan en tres, a saber: padres que poseen hasta estudios primarios, padres con estudios medios (uno de ellos o ambos) y padres con estudios superiores (uno de ellos o ambos).

Con respecto a la variable ocupación, nuestro trabajo se centra en considerar el nivel ocupacional más alto, ya sea del padre o de la madre (Fachelli, 2010). De este modo diferenciamos grupos de ocupados y, concretamente, seleccionamos el máximo estatus ocupacional del padre o de la madre con el fin de analizar las características principales de la familia de los universitarios. De esta manera, la clasificación que proponemos queda establecida en cinco categorías, según se detalla a continuación:

1. Trabajo por cuenta ajena: dirección.

2. Trabajo por cuenta ajena: técnico superior (que incluye trabajo por cuenta propia que requiere estudios universitarios).

3. Trabajo por cuenta ajena: cualificado.

4. Trabajo por cuenta propia: no requiere estudios universitarios.

5. Trabajo por cuenta ajena: no cualificado.

En general, los análisis resultantes de este tipo de organización de la información (matrices de transición) son más interesantes cuantas más categorías posee la información de base. Por esa razón, cuando utilicemos estas matrices, mantendremos estas cinco categorías. Asimismo, decidimos seguir el mismo procedimiento con los hijos, es decir, reorganizando la información de igual manera que la encuesta recoge la ocupación de los padres ${ }^{4}$. Para evitar la dificultad de comparar entre los titulados que trabajan a tiempo parcial y los que lo hacen a tiempo completo, en el momento de pasar la encuesta, seleccionamos a los titulados ocupados que realizan tareas a tiempo completo, los cuales suman el $80 \%$ de la muestra total.

\section{1. ¿Dé qué universidades hablamos?}

La distribución entre alumnos universitarios del sector público y privado en Cataluña es de un $90 \%$ en el sector público y un 10\% en el privado. La encuesta en que se basa este artículo no abarca a los graduados de todas las universidades del Principado, en primer lugar, porque se ha considerado que los de la Universitat Oberta de Catalunya (que representan el 18\% de los estudiantes),

4. En el Anexo 1 se presenta la ficha técnica de la encuesta llevada a cabo por AQU. 
dadas sus peculiares características de educación a distancia, no podían analizarse conjuntamente con los de las universidades presenciales. En segundo lugar, porque la encuesta no ha podido entrevistar a los graduados de tres universidades privadas, que representan el $7 \%$ del alumnado universitario.

Debido a ello, la población de referencia de este análisis está constituida por los graduados de todas las universidades públicas presenciales y una de las privadas, que representan el $25 \%$ de los estudiantes del sector privado. Estas universidades acogen al 75\% del total de los universitarios catalanes.

Si queremos hacer una tipología comprensible de las diferencias entre las universidades, más allá de la señalada entre pública y privada, podemos establecer tres ejes: a) histórico; $b$ ) ubicación geografía y área de influencia, y c) orientación i/o vocación.

La encuesta incluye a las dos universidades «históricas» (anteriores al año 1968) que hay en Cataluña: la Universitat de Barcelona (UB) y la Universitat Politècnica de Catalunya (UPC); una universidad pública creada en 1968: la Universitat Autònoma de Barcelona (UAB); las que emergieron a partir de la descentralización de la oferta universitaria en la década de 1980 y las de creación más reciente: la pública Universitat Pompeu Frabra (UPF) y la privada Universitat de Vic (UV).

Respecto a su ubicación, cuatro de las universidades consideradas se hallan en el Área Metropolitana de Barcelona y escolarizan al 71\% del alumnado universitario "presencial» de Cataluña, mientras que las universidades "presenciales» situadas fuera del área de la Ciudad Condal (llamémoslas «descentralizadas»), aunque solo representan al 20\% del alumnado, han desempeñado un papel clave en el crecimiento y la democratización de la población universitaria del Principado, al disminuir, entre otros, los costos indirectos de los estudios superiores, derivados del cambio de residencia para que los jóvenes que viven fuera del Área Metropolitana de Barcelona puedan acceder a la universidad.

\section{Resultados}

\subsection{Una aproximación a la equidad: el nivel de estudios de los padres ${ }^{5}$, de la generación de los padres y su presencia entre los graduados}

Un primer dato de carácter global es que un porcentaje muy importante de los titulados procede de hogares donde los padres tienen como máximo estudios primarios (un 40,1\%), el resto se reparte aproximadamente en partes iguales entre los procedentes de hogares con padres que tienen estudios medios y superiores.

5. Utilizamos como indicador del origen social de los graduados el nivel de estudios de los padres, porque, en las investigaciones recientes, es el factor de mayor influencia en la demanda de educación universitaria. Para España, ver los artículos de Laura de Pablos y María Gil (2006), Marta Rahona (2009) y Cecilia Albert (2000). 
Tabla 1. Máximo nivel de estudios de los padres de los titulados universitarios

\begin{tabular}{lcclc}
\hline Máximo nivel de estudios de los padres & Casos & $\%$ & $\begin{array}{c}\text { Agrupación en } \\
\text { tres categorías }\end{array}$ & $\%$ \\
\hline $\begin{array}{l}\text { Los dos tienen estudios primarios } \\
\text { o no tienen estudios }\end{array}$ & 4.908 & 40,1 Primarios & 40,1 \\
Uno de los dos tiene estudios medios & 1.828 & 14,9 Con estudios & \\
Los dos tienen estudios medios & 1.918 & 15,7 medios & 30,6 \\
Uno de los dos tiene estudios superiores & 2.054 & 16,8 Con estudios & \\
Los dos tienen estudios superiores & 1.524 & 12,5 superiores & 29,3 \\
Total & 12.232 & 100,0 Total & 100,0 \\
\hline
\end{tabular}

Fuente: elaboración propia sobre la base de AQU (2008).

Pero esta distribución es moderadamente heterogénea si la consideramos según universidades, itinerarios formativos y simultaneidad entre estudio y trabajo (para ampliar este aspecto, ver Planas y Fachelli, 2010).

Un elemento importante a destacar es que, en 2004, se graduaron más mujeres que varones (un $61 \%$ versus un $39 \%$ ). Si reprodujéramos la tabla 1 diferenciada según el sexo de los graduados, las proporciones se mantienen prácticamente igual entre los hijos y las hijas de padres que tienen estudios primarios, y se modifican levemente en las dos categorías siguientes. Los hijos varones de padres con estudios medios representan el 29,3\%, mientras que las hijas son el 31,5\%. Cuando los padres tienen estudios superiores, la diferencia entre el sexo de los hijos aumenta: los varones representan el 31,4\%, mientras que las mujeres conforman el $27,9 \%$.

La encuesta no proporciona la edad de los padres de los graduados, por ello hemos realizado una estimación. El objetivo es disponer de un indicador de equidad en la obtención de la titulación universitaria, puesto que nos interesa analizar la relación de los padres de los titulados con respecto al promedio de la población de su generación. Para ello, hemos realizado un ejercicio que es orientativo sobre la relación entre los padres de los alumnos que se han titulado y su generación, con respecto al nivel de estudios, observando la edad de los padres en encuestas vitales. El resultado es que la generación "promedio» de los padres de los graduados en 2004 en Cataluña es la de los padres nacidos en 1949 y las madres nacidas en 1953.

En función de estos datos, podemos plantear una relación que nos ilustraría el nivel de representatividad de los padres de los titulados universitarios en relación con su generación. Esta relación se presenta en el gráfico 1 .

Cabe destacar que el índice de presencia refleja la comparación del nivel educativo de la generación que tiene entre 55 y 59 años con el grupo de padres de los titulados universitarios. Así, el valor 1 implicaría igualdad de presencia de los diferentes niveles de estudios de los padres de universitarios en relación con el de su generación; un valor inferior o superior a 1 de los grupos bajo análisis implica subrepresentación o sobrerrepresentación, respectivamente. 
Gráfico 1. Comparación del nivel educativo de los padres de los titulados en 2004 con el nivel educativo de su generación (personas entre 55 y 59 años de edad). Índice de presencia

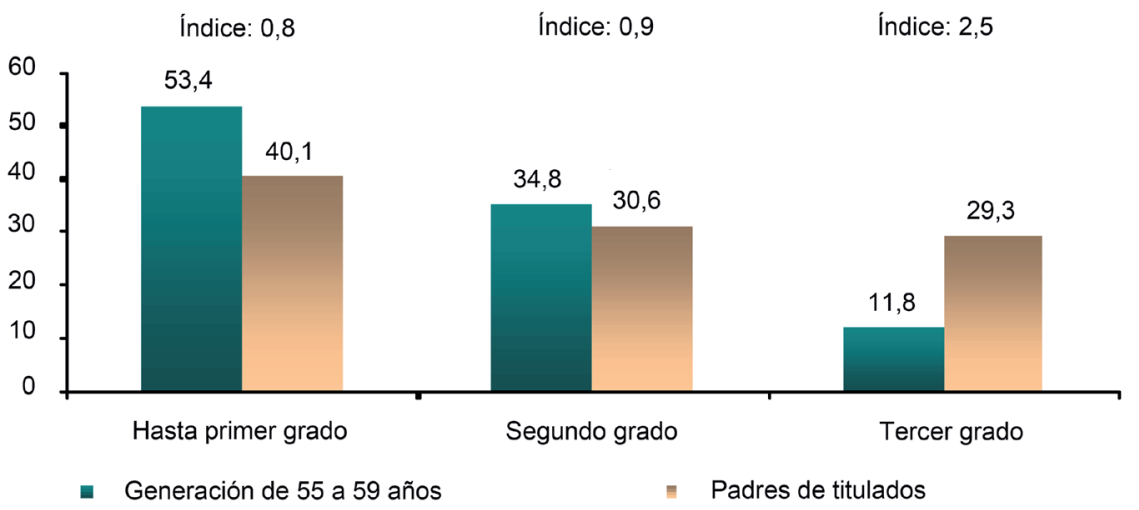

Fuente: microdatos de la encuesta de AQU (2008) y de la EPA (segundo semestre de 2005).

Observamos que los padres sin estudios o con estudios primarios que tienen a sus hijos en la universidad están levemente subrepresentados en la universidad en relación con su generación $(0,8)$. Los padres con estudios medios estarían representados en la universidad casi en forma proporcional a su generación $(0,9)$. Finalmente, los padres de titulados con estudios superiores están sobrerrepresentados en la universidad $(2,5)$, es decir, la proporción de titulados superiores con hijos que se titularon en 2004 es netamente superior a la presencia en su generación, pues solamente el 11,8\% de las personas entre 55 y 59 años tiene estudios superiores y, dentro de la universidad, este porcentaje llega a casi al 30\%.

Presentando los mismos datos de forma tal que nos indiquen las probabilidades de ser titulado universitario según los niveles de estudios de los padres, si la probabilidad promedio de ser titulado universitario de la generación nacida en el año 1980 es del 28\% (EPA del segundo trimestre de 2009), las probabilidades de titularse en la universidad en función de los niveles de estudios de los padres, obtenidas de multiplicar esta cifra promedio por el índice de presencia de cada grupo de padres (gráfico 1), son las siguientes:

Tabla 2. Porcentaje de hijos titulados universitarios y no universitarios según el nivel de estudios de los padres para el conjunto de la generación de los graduados

\begin{tabular}{llcccr}
\hline Padres & & Primario & Secundario & Universitario & \multicolumn{1}{c}{ Total } \\
\hline \multirow{2}{*}{ Titulación } & Universitarios & $22,4 \%$ & $25,2 \%$ & $70,0 \%$ & $28,0 \%$ \\
de los hijos & No universitarios & $77,6 \%$ & $74,8 \%$ & $30,0 \%$ & $72,0 \%$ \\
& Total & $100,0 \%$ & $100,0 \%$ & $100,0 \%$ & $100,0 \%$ \\
\hline
\end{tabular}

Fuente: elaboración propia a partir de microdatos de la encuesta de AQU (2008) y de la EPA (segundo trimestre de 2005 y segundo trimestre de 2009). 
Pero este fenómeno no ha sido siempre así, sino que, como muestra Marina Subirats en base a la encuesta metropolitana de Barcelona (2009: 19-20), es el resultado de un proceso gradual de incremento de la participación de las «categorías profesionales bajas» en la universidad.

A partir de los datos presentados anteriormente, encontramos cuatro hechos que merecen la pena ser destacados:

1) La gran mayoría de los titulados universitarios en 2004 son hijos de personas que no tienen estudios universitarios (un 70\%).

2) Entre éstos, los graduados que provienen de familias con un nivel educativo primario o inferior constituyen la mayoría relativa (un $40 \%$ del total).

3) Hay muy pocas diferencias en las oportunidades de acceso a las titulaciones universitarias entre los hijos que provienen de familias con estudios primarios o menos y de aquellos que provienen de familias con estudios secundarios.

4) Los hijos de titulados universitarios, aunque constituyen una minoría en la universidad actual, tienen muchas más oportunidades de acceder a la universidad, que los que provienen de hogares con padres sin estudios superiores.

Finalmente, es importante señalar el carácter aproximativo que tiene este ejercicio, pues la comparación se realiza entre una estimación sobre el nivel de estudios de la generación de los padres y la información que los hijos nos dan en la encuesta AQU sobre el nivel educativo más alto de la madre o del padre.

\section{2. ¿Cuál es la ocupación de los graduados en comparación con la de sus padres?}

Antes que nada, queremos resaltar la alta tasa de ocupación de los titulados, pues la tasa de desocupación en 2008 de los titulados de las universidades catalanas en 2004 era sólo del 3,1\% (AQU, 2008). Estas cifras dan la razón a Manel Castells (en la presentación del libro de Carnoy) cuando afirma que:

[...] uno de los errores más crasos que se repiten en los medios de comunicación en nuestro país es aquello de que «la universidad es una fábrica de parados». $\mathrm{Ni}$ es así en España (la tasa de paro de los universitarios es muy inferior a la de los que no pasan de secundaria) ni en el mundo, como ha demostrado Martin Carnoy, al encontrar una alta correlación estadística entre el número de años de estudio y el nivel de salarios a lo largo de la carrera de una persona. (Carnoy, 2006: 15)

Para bordar la comparación entre la ocupación de los graduados y la de sus padres, reagrupamos cinco categorías ocupacionales en tres grupos, igual que lo hemos hecho con el nivel de estudios de los padres, con el fin de lograr una mayor capacidad descriptiva y expositiva del origen familiar de los titulados universitarios en función de la jerarquía ocupacional de sus progenitores. En 
Tabla 3. Máximo nivel ocupacional de los padres

\begin{tabular}{|c|c|c|c|c|}
\hline Máximo nivel ocupacional de los padres & Casos & $\%$ & $\begin{array}{l}\text { Estatus } \\
\text { ocupacional }\end{array}$ & $\%$ \\
\hline Trabajo por cuenta ajena: dirección & 1.894 & 15,7 & & \\
\hline Trabajo por cuenta ajena: técnico superior & 1.523 & 12,6 & & \\
\hline $\begin{array}{l}\text { Trabajo por cuenta propia: } \\
\text { requiere estudios universitarios }\end{array}$ & 840 & 7,0 & Estatus alto & 35,3 \\
\hline Trabajo por cuenta ajena: cualificado & 3.849 & 31,9 & Estatus medio & 31,9 \\
\hline $\begin{array}{l}\text { Trabajo por cuenta propia: } \\
\text { no requiere estudios universitarios }\end{array}$ & 2.493 & 20,7 & & \\
\hline Trabajo por cuenta ajena: no cualificado & 1.453 & 12,1 & Estatus bajo & 32,7 \\
\hline Total & 12.052 & 100,0 & Total & 100,0 \\
\hline
\end{tabular}

Fuente: elaboración propia sobre la base de AQU (2008).

primer lugar, presentamos, en la tabla 3, el máximo nivel ocupacional de los padres.

La distribución del estatus ocupacional de los padres de los titulados se agrupa en tres partes casi iguales. Esta distribución presenta una variación relativa al considerarla según universidades, itinerarios formativos y simultaneidad entre estudio y trabajo (para ver estos aspectos con mayor detalle, consultar Planas y Fachelli, 2010).

En términos globales, la tabla 4 pone de manifiesto la relación entre la ocupación de los hijos y la de los padres. Así, podemos observar la proporción de titulados que realizan tareas del mismo nivel y también aquellos que realizan tareas de nivel diferente al de sus padres, ya sea de mayor jerarquía o de menor jerarquía. El resultado que se presenta a continuación refleja a todos los titulados ocupados a tiempo completo.

Una gran parte de los titulados universitarios, pese a que se encuentra en su primera inserción profesional, ya llega a posiciones más elevadas que las que realizan sus padres, que, debido a su edad, se encuentran en la fase final de su carrera profesional. El 51,1\% del total de titulados (que se obtiene de sumar

Tabla 4. Ocupación del titulado según la ocupación de sus padres

\begin{tabular}{|c|c|c|c|c|c|}
\hline \multirow[b]{2}{*}{$\begin{array}{l}\text { Máximo estatus } \\
\text { ocupacional de los padres }\end{array}$} & \multicolumn{5}{|c|}{ Titulados universitarios ocupados a tiempo completo } \\
\hline & Dirección & $\begin{array}{l}\text { Técnico } \\
\text { superior }\end{array}$ & Cualificado & $\begin{array}{l}\text { Cuenta } \\
\text { propia }\end{array}$ & $\begin{array}{l}\text { No } \\
\text { cualificado }\end{array}$ \\
\hline 1. Dirección & 6,0 & 1,8 & 6,8 & 0,7 & 0,3 \\
\hline 2. Técnico superior & 6,2 & 2,4 & 8,6 & 1,1 & 0,6 \\
\hline 3. Cualificado & 9,5 & 4,4 & 15,5 & 1,0 & 1,3 \\
\hline $\begin{array}{l}\text { 4. Cuenta propia sin } \\
\text { estudios universitarios }\end{array}$ & 6,4 & 2,9 & 10,0 & 1,3 & 0,8 \\
\hline 5. No cualificado & 3,7 & 1,3 & 6,3 & 0,4 & 0,7 \\
\hline
\end{tabular}

Fuente: elaboración propia sobre la base de AQU (2008). 
Tabla 5. Ocupación de titulados por género según ocupación de sus padres

\begin{tabular}{|c|c|c|c|c|c|c|c|c|c|c|}
\hline \multirow{2}{*}{$\begin{array}{l}\text { Máximo estatus } \\
\text { ocupacional de los padres }\end{array}$} & \multicolumn{5}{|c|}{ Mujeres tituladas ocupadas } & \multicolumn{5}{|c|}{ Varones titulados ocupados } \\
\hline & 1 & 2 & 3 & 4 & 5 & 1 & 2 & 3 & 4 & 5 \\
\hline 1. Dirección & 4,8 & 1,8 & 7,2 & 0,5 & 0,4 & & 9 & & & \\
\hline 2. $\mathrm{T}$ & 5,5 & 2,0 & 9 , & & 0,8 & 7,3 & 3,0 & 7,3 & & 04 \\
\hline 3. Cua & 8,3 & 4,4 & 17,4 & 0,7 & 1,6 & 11,3 & 4,3 & 12,7 & 1,4 & 1,0 \\
\hline $\begin{array}{l}\text { 4. Cue } \\
\text { estu }\end{array}$ & 5,9 & $2, j$ & 11, & 1,1 & 0,0 & $/, 1$ & 2,0 & 0,1 & 1,5 & 0,8 \\
\hline 5. No cualificado & 3,5 & 1,2 & 6,7 & 0,3 & 0,7 & 4,0 & 1,4 & 5,6 & 0,5 & 0,7 \\
\hline
\end{tabular}

Fuente: elaboración propia sobre la base de AQU (2008).

todos los porcentajes del margen izquierdo e inferior del cuadro) ya realizan tareas superiores en jerarquía a las de sus padres.

Por su parte, el $25,9 \%$ realiza tareas similares a las que realizan sus padres (suma de la diagonal) y el $23,3 \%$ todavía realiza tareas inferiores en jerarquía (margen derecho y superior del cuadro).

Es importante destacar que la inserción de los titulados que estamos analizando es temprana, con lo cual nos queda un largo camino por delante en su carrera profesional y, en muchos casos, este hecho implicará un ascenso ocupacional de los titulados, que tenderán, en una proporción todavía mayor a la actual, a superar las posiciones ocupacionales de sus padres.

Si la observación se realiza por género, resulta que los hombres llevan a cabo tareas de mayor jerarquía que las mujeres, aunque las diferencias sean pequeñas (un $52,4 \%$ y un $49,8 \%$, respectivamente) (tabla 5 ).

Consecuentemente, las mujeres están sobrerrepresentadas en tareas de menor jerarquía. Por ejemplo: los hombres que efectúan tareas de dirección con respecto a sus padres, que son técnicos superiores o trabajadores cualificados, suman un 18,6\%, en cambio, en el caso de las mujeres, esta cifra es de un $13,7 \%$.

Por otro lado, si se observan las ocupaciones por encima de la diagonal (tareas de nivel inferior que la que realizan sus padres), las mujeres representan en conjunto el $24,2 \%$, en cambio, los hombres constituyen el $22 \%$.

La proporción de mujeres y hombres que desarrolla tareas del mismo nivel que sus padres es similar (el 26\% y el 25,6\%, respectivamente). En ambos casos, la mayor tasa se encuentra en las tareas cualificadas (el 17,4\% para las mujeres y el $12,7 \%$ para los hombres). Finalmente, los hombres tienen mayor presencia en los lugares más altos que las mujeres (el 10,6\% contra el 6,8\% en lugares de dirección y técnico superior).

\subsection{Ocupación de los hijos en relación con la ocupación de los padres}

Si realizamos una lectura considerando la ocupación del padre como indicador de origen familiar del titulado universitario, obtenemos los resultados que aparecen en la tabla 6. 
Tabla 6. Ocupación de los titulados según la ocupación de los padres

\begin{tabular}{|c|c|c|c|c|c|c|}
\hline \multirow{2}{*}{$\begin{array}{l}\text { Máximo estatus } \\
\text { ocupacional } \\
\text { de los padres }\end{array}$} & \multicolumn{6}{|c|}{ Titulados universitarios ocupados a tiempo completo } \\
\hline & Dirección & $\begin{array}{l}\text { Técnico } \\
\text { superior }\end{array}$ & Cualificado & $\begin{array}{l}\text { Cuenta } \\
\text { propia }\end{array}$ & $\begin{array}{l}\text { No } \\
\text { cualificado }\end{array}$ & Total \\
\hline 1. Dirección & 38,0 & 11,7 & 43,4 & 4,8 & 2,2 & 100 \\
\hline 2. Ténico superior & 32,6 & 12,7 & 45,4 & 6,0 & 3,3 & 100 \\
\hline 3. Cualificado & 30,0 & 13,7 & 49,0 & 3,2 & 4,2 & 100 \\
\hline $\begin{array}{l}\text { 4. Cuenta propia } \\
\text { sin estudios } \\
\text { universitarios }\end{array}$ & 30,0 & 13,4 & 46,7 & 6,0 & 3,9 & 100 \\
\hline 5. No cualificado & 29,8 & 10,3 & 51,0 & 3,0 & 5,9 & 100 \\
\hline
\end{tabular}

Fuente: elaboración propia sobre la base de AQU (2008).

1. Dirección: teniendo en cuenta que esta es la categoría ocupacional más alta $\mathrm{y}$, consecuentemente, no puede tener movilidad ascendente, se observa que el 38\% de los hijos realiza tareas del mismo nivel, el $43,4 \%$ realiza tareas cualificadas y el $11,7 \%$, un trabajo de técnico superior. Muy pocos hijos se ocupan como cuenta propia (el $4,8 \%$ ) y solamente el $2,2 \%$ realiza un trabajo no cualificado.

2. Técnico superior: casi el $13 \%$ de los hijos realiza tareas del mismo nivel que sus padres, mientras que alrededor de un tercio los supera jerárquicamente, pues desarrolla tareas de dirección. Por el contrario, el $45 \%$ realiza tareas cualificadas y el resto se reparte entre el $6 \%$ de trabajo por cuenta propia y el $3,3 \%$ en tareas no cualificadas.

3. Cualificado: casi el $50 \%$ de los hijos realiza tareas del mismo nivel que sus padres. Por su parte, el 43,7\% supera a sus padres en jerarquía, mientras que poco más de un $7 \%$ realiza tareas de menor jerarquía.

4. Cuenta propia (sin estudios universitarios): los hijos que realizan el mismo nivel de tarea que sus padres, en este caso, disminuye al 6\%. Excluyendo casi a un $4 \%$ que realiza tareas no cualificadas, el $90 \%$ restante se ocupa de tareas de mayor jerarquía.

5. No cualificado: el $6 \%$ de los hijos realiza tareas del mismo nivel que sus padres y, dado que esta es la categoría ocupacional más baja, el resto realiza tareas de mayor jerarquía.

Si realizamos el mismo análisis teniendo en cuenta el sexo de los titulados, aparecen diferencias relevantes. Del total de hijos hombres de padres directores, el $44 \%$ ocupa el mismo lugar y este porcentaje se reduce al $33 \%$ en el caso de las mujeres. Esta situación se compensa con una mayor proporción de mujeres en lugares cualificados (el 50\% versus el 36\% de los hombres).

Los hijos varones de los técnicos superiores realizan tareas de dirección en mayor medida (un 37,5\%) que las mujeres del mismo origen familiar (un $29,2 \%)$. Los hombres que coinciden con el lugar de origen de sus padres son más del 15\%, mientras que las mujeres son aproximadamente el 10\%. 
Tabla 7. Ocupación de los titulados por género, según ocupación de origen de los padres

\begin{tabular}{lrrrrrrr}
\hline \multirow{2}{*}{$\begin{array}{l}\text { Máximo estatus ocupacional } \\
\text { de los padres }\end{array}$} & \multicolumn{7}{c}{ Mujeres tituladas ocupadas } \\
\cline { 2 - 8 } & $\mathbf{1}$ & $\mathbf{2}$ & $\mathbf{3}$ & $\mathbf{4}$ & 5 & Total \\
\hline 1. Dirección & 32,9 & 12,1 & 49,2 & 3,3 & 2,5 & 100 \\
2. Técnico superior & 29,2 & 10,7 & 51,2 & 4,7 & 4,2 & 100 \\
3. Cualificado & 25,6 & 13,5 & 53,8 & 2,3 & 4,8 & 100 \\
4. Cuenta propia sin estudios universitarios & 27,0 & 13,1 & 50,9 & 5,2 & 3,8 & 100 \\
5. No cualificado & 28,1 & 9,7 & 54,3 & 2,2 & 5,8 & 100 \\
\hline Máximo estatus ocupacional & \multicolumn{7}{c}{ Varones titulados ocupados } \\
\cline { 2 - 8 } de los padres & 1 & 2 & 3 & 4 & 5 & Total \\
\hline 1. Dirección & 44,4 & 11,1 & 36,1 & 6,5 & 1,8 & 100 \\
2. Técnico superior & 37,5 & 15,5 & 37,2 & 7,7 & 2,1 & 100 \\
3. Cualificado & 36,7 & 14,0 & 41,5 & 4,6 & 3,2 & 100 \\
4. Cuenta propia sin estudios universitarios & 34,8 & 13,8 & 40,0 & 7,2 & 4,2 & 100 \\
5. No cualificado & 32,4 & 11,3 & 46,0 & 4,2 & 6,1 & 100 \\
\hline
\end{tabular}

Fuente: elaboración propia sobre la base de AQU (2008).

Los hijos de trabajadores cualificados que realizan las mismas tareas que sus padres son más en el caso de las mujeres (un 53,8\%), que en el de los hombres (un 41,5\%).

Si observamos la categoría de los padres que se ocupan por cuenta propia sin estudios universitarios, vemos que el porcentaje de hijos que los superan en otras ocupaciones de mayor jerarquía es similar entre hombres (un 89\%) y mujeres (un 91\%).

Los hijos que realizan la misma tarea, no cualificada, que sus padres son alrededor del 6\% en ambos sexos y es la categoría más baja. El 94\% restante ocupa categorías cualificadas.

Finalmente, parece razonable concluir que la universidad estaría dando herramientas para el posicionamiento de los hijos en lugares que superan jerárquicamente la tarea que realizan sus padres y que la influencia del origen de los padres en términos ocupacionales sobre la ocupación del hijo no es muy importante.

\section{Conclusiones}

Recapitulando, los principales resultados presentados en este texto son dos:

a) Sin llegar a una equidad total, pues los hijos de titulados universitarios están sobrerrepresentados entre los titulados universitarios, siete de cada diez titulados son la primera generación que accede a la universidad de su familia y el coeficiente de presencia de los titulados con familias con nivel de estudios primarios y secundarios son, respectivamente, 0,8 y 0,9 , lo que se aproxima bastante al 1 que significaría la equidad. 
b) El segundo resultado que queremos subrayar es la elevada movilidad ocupacional ascendente de los titulados respecto de sus padres, y recordemos que se trata de una comparación entre una generación, la de los padres, que se encuentra al final de su carrera profesional, y otra, la de los hijos, que la ha iniciado recientemente. Observamos que el sistema universitario está facilitando que sus titulados se sitúen mayoritariamente, tan solo a cuatro años de haber acabado sus estudios, en ocupaciones jerárquicamente superiores a las de sus padres. Esto no quiere decir necesariamente que la ocupación en que trabajan satisfaga todas las expectativas de los nuevos titulados, pero nos hace pensar que se aproxima, dado que el porcentaje de aquellos que consideran su inserción adecuada con respecto a su formación es el $80 \%$ y que el conjunto de los encuestados valora la formación recibida globalmente con un «7/10 puntos».

En estas conclusiones, prestaremos especial atención al análisis de dos fenómenos relacionados con estos resultados: en primer lugar, al comportamiento del sistema educativo como institución que ha «encuadrado» y ha hecho posible los cambios en la presencia de estudiantes y graduados universitarios provenientes de familias de distinto origen social; en segundo lugar, a la movilidad intergeneracional observada entre los graduados y sus padres, con el fin de valorar en qué medida esta se corresponde con una movilidad neta o meramente estructural.

Respecto al primer aspecto, en las primeras páginas de este texto, planteábamos una pregunta respecto a la equidad de nuestros sistemas educativos: $¿ \mathrm{La}$ escuela, en este caso la universidad, es una institución social que proporciona las mismas oportunidades a todos los jóvenes independientemente de su familia de origen, o, por el contrario, refuerza y legitima las desigualdades derivadas del origen social de los estudiantes?

La respuesta, como también indican otros autores (Rahona, 2009), y vistos los resultados presentados, no puede inclinarse categóricamente por ninguna de las dos opciones sin matices, pero del análisis de los datos que hemos realizado, se desprenden dos corolarios particularmente relevantes respecto a la función social de las universidades catalanas. En primer lugar, que se observan adelantos en la igualdad de las oportunidades en la obtención de las titulaciones en las universidades catalanas consideradas globalmente, lo que nos permite presuponer que la mencionada equidad también se da en el acceso a las universidades. En consecuencia, debemos destacar que el sistema universitario catalán, en su tarea de elevar el nivel de estudios de la población, también ha elevado la equidad en su acceso, aunque sin alcanzarla plenamente, como subraya Rahona (2009). Este hecho, por otra parte, coincide con los datos presentados por la OCDE (2007) en referencia al conjunto de las universidades españolas en comparación con las de otros países pertenecientes a la misma organización internacional. De todas maneras, como señala Calero (2006), de los resultados obtenidos acerca de los graduados catalanes, la diversidad en las oportunidades de acceso a la educación entre las distintas comunidades autónomas no permite inferir conclusiones válidas para el conjunto de España. 
El fenómeno observado, de presencia mayoritaria y proporcionalmente creciente de padres no universitarios entre los titulados de las universidades catalanas, como hemos indicado al principio de este texto, es el resultado de un consenso entre tres tipos de instituciones: las familias, el mercado de trabajo y el estado, que han apoyado este crecimiento de la educación y de la equidad en el acceso a la universidad.

$\mathrm{Si}$ analizamos los principales cambios en el sistema educativo que han acompañado a los que han tenido lugar en la equidad en el acceso a la universidad y posterior graduación han sido - a partir de nuestro informe anterior (Planas y Fachelli, 2010)—, en primer lugar, el crecimiento de la oferta de plazas universitarias que se ha producido, particularmente durante el periodo democrático. Pero este crecimiento no explicaría los efectos producidos sobre el acceso a la universidad si no lo relacionamos con la descentralización de esta oferta que ha acercado a la población externa al área de Barcelona, reduciendo los costes indirectos de los estudios universitarios para esta población y en universidades que imparten títulos jerárquicamente equivalentes a los del área de Barcelona.

Un papel clave ha tenido también la reestructuración de ciclos del sistema universitario ya formulado en la LGE, puesto que ha incorporado ciclos cortos y ha establecido su conexión, y continuidad, con los de licenciatura. Este cambio ha tenido un efecto en el acceso a la universidad de estudiantes con origen social inferior que han privilegiado esta vía respecto de los ciclos largos de licenciatura, como muestran los resultados presentados en nuestro estudio previo. También hemos constatado que los estudiantes que trabajan durante sus estudios pertenecen mayoritariamente a familias con padres de bajo nivel de estudios.

Por último, la apertura del acceso a la universidad a los estudiantes provenientes de CFGS (ciclos formativos de grado superior) ha aumentado las oportunidades de acceso de este mismo tipo de estudiantes, como lo muestran los datos elaborados por Planas et al. (2011).

Ello plantea interrogantes importantes respecto a los efectos de una serie de medidas recientes, y en mayor o menor medida asociadas al llamado "Proceso de Bolonia», que, según lo anteriormente dicho, son potencialmente regresivos sobre la oportunidad de acceso y titulación universitaria de los hijos con padres con niveles inferiores.

Por un lado, la jerarquización de las universidades en Cataluña, mediante la competición por la excelencia, está dejando grosso modo en un segundo lugar a las universidades de fuera del área de Barcelona. Por otro lado, la sustitución de los ciclos cortos en nuestras universidades por los "grados", de cuatro años, podría tener efectos sobre la equidad, puesto que, como se mencionó anteriormente, los ciclos cortos han beneficiado en mayor medida a los hijos provenientes de familias con menor nivel socioeconómico.

En tercer lugar, establecer un tipo de organización de la enseñanza universitaria que presupone que los estudiantes lo son a tiempo completo, puede excluir o limitar el acceso a aquellos estudiantes provenientes de un nivel social más bajo que estudian y trabajan con mayor frecuencia. 
Finalmente, medidas que puedan frenar el acceso de estudiantes universitarios provenientes de la formación profesional pueden tener también un efecto restrictivo del acceso a la universidad de los universitarios con un nivel social más bajo (Planas et al., 2011)

Respecto a los resultados observados referentes a la movilidad intergeneracional, la pregunta que se plantea es: ¿En qué medida esta movilidad refleja movilidad social de los jóvenes titulados universitarios o movilidad estructural debida simplemente a los cambios en la estructura del empleo entre el momento de inserción profesional de los titulados universitarios y el de sus padres?

No podemos responder a la pregunta referente a la movilidad social, porque no disponemos de datos equivalentes respecto del conjunto de sus coetáneos y, por otra parte, podemos afirmar sin dudas que la estructura del empleo en el momento de inserción de los hijos es muy distinta de la de los padres. De todas maneras, podemos establecer dos hipótesis extremas, que, por esta razón, son poco verosímiles y nos permiten afirmar que los hechos se sitúan en un punto intermedio que, por falta de datos, no podemos precisar.

La primera hipótesis es que la movilidad intergeneracional es un reflejo bastante fiel de una movilidad social entre los hijos, para ello no se deberían haber producido cambios en la estructura social entre las dos generaciones, hipótesis poco plausible, por lo cual podemos afirmar que la movilidad intergeneracional no refleja en su totalidad una movilidad neta entre los jóvenes titulados.

La segunda hipótesis, opuesta a la anterior, es que la movilidad intergeneracional refleja simplemente los cambios en la estructura de empleo que se ofrece a cada generación. En el caso de que fuera así, supondría que los titulados universitarios han accedido al empleo adecuándose de manera extraordinariamente exacta a los cambios en la estructura de empleo, lo cual, en sí mismo, ya sería un importante resultado que mostraría una fiel adecuación entre la producción de titulados universitarios y los cambios en la demanda de empleo.

La hipótesis más razonable es que nos hallamos en una situación intermedia donde la movilidad intergeneracional refleja simultáneamente movilidad neta y estructural en proporciones que no podemos establecer con la información disponible y que podría ser el objeto de futuras investigaciones.

\section{Referencias bibliográficas}

AlberT, C. (2000). «Higher education demand in Spain: The influence of the labour market signals and family background». Higher Education, 40, 147-162.

AQU (2003). Educació superior i treball a Catalunya: Estudi de la inserció laboral dels graduats de les universitats públiques catalanes. Barcelona: Agència per a la Qualitat del Sistema Universitari de Catalunya.

- (2007). Educació superior i treball a Catalunya: Anàlisi dels factors d’inserció laboral. Barcelona: Agència per a la Qualitat del Sistema Universitari de Catalunya. 
AQU (2008). Tercer estudi d'inserció laboral dels graduats de les universitats catalanes: Primera valoració dels resultats. Barcelona: Agència per a la Qualitat del Sistema Universitari de Catalunya.

Baudelot, C. y Establet, R. (1979). L'école capitaliste en France. París: Maspero.

BÉDUwÉ, C. y PlanAs, J. (2003). Educational Expansion and Labour Market-EDEX. Luxemburgo: Office for Official Publications of the European Communities.

Blau, P. y Duncan, O. (1967). The American Occupational Structure. Nueva York: John Wiley and Sons.

Boudon, R. (1983). La desigualdad de oportunidades. Barcelona: Laia.

BOWLES, S. y GINTIS, H. (1976). Schooling in capitalista America: Educational reform and contradictions of economic life. Nueva York: Basic Books.

CALERo, J. (2006). La equidad en educación: Informe analítico del sistema educativo español. Madrid: CIDE.

CARABAÑA, J. (1999). Dos estudios sobre movilidad intergeneracional. Madrid: Fundacion Argentaria.

Castells, M. (2006). «Presentación». En: Carnoy, M. Economía de la educación. Barcelona: UOC.

Coleman, J. (1979). Equality of Educational Opportunity. Nueva York: Arno Press. (Versión original de 1966.)

- (1982). The Asymmetric Society. Syracuse University Press.

Consell Superior D’Avaluació Del Sistema Educatiu (2008). PISA 2006: Resultats de l'alumnat de Catalunya: Avaluació de l'educació secundària obligatòria. Barcelona: Generalitat de Catalunya.

CROMPton, R. (1999). Restructuring gender relations and employment: The decline of the male breadwinner. Oxford: Oxford University Press.

esteban, F.; Martín, A.; Miguélez, F.; Molina, O. y Recio, A. (2009). Crisi i desocupació: Quines polítiques? Barcelona: Associació Catalana de Sociologia. Filial de l'Institut d'Estudis Catalans.

European Ministers Responsible for Higher Education (2009). The Bologna Process 2020: The European Higher Education Area in the new decade. Leuven and Louvain-la-Neuve: Communiqué of the Conference.

[http://www.ond.vlaanderen.be/hogeronderwijs/bologna/conference/documents/Leuven_Louvain-la-Neuve_Communiqué_April_2009.pdf]

FACHELLI, S. (2010). Nuevo modelo de estratificación social y nuevo instrumento para su medición: El caso argentino. Tesis doctoral inédita. Barcelona.

GOLDTHORPE, J. y JACKSON, M. (2007). «Intergenerational class mobility in contemporary Britain: political concerns and empirical findings». The British Journal of Sociology, 58 (4), 525-546.

GoldthorPe, J.; Llewellyn, C. y Payne, C. (1980). Social Mobility \& Class Structure in Modern Britain. Oxford: Clarendon Press.

Hartog, J. (2000). «Human Capital as an Instrument of Analysis for the Economics of Education». European Journal of Education, 35 (1), 7-20.

HERNÁNDEZ DE FRUTOS, T. (1997). Las estructuras sociales. Madrid: Verbo Divino.

Hillage, J. y Pollard, E. (1998). Employability: Developing a framework for policy analysis. Londres: Department for Education and Employment (DfEE). Research report RR85.

Hitlin, S. (2006). «Parental influences on children's values and aspirations: Bridging two theories of social class and socialization». Sociological Perspectives, 49 (1), $25-46$. 
Institut D'Estadística de CATAlunya (IDESCAT). Estadistica bàsica de Catalunya: Demografia $i$ qualitat de vida. [http://www.idescat.cat/ dequavi/?TC $=444 \& \mathrm{~V} 0=4 \& \mathrm{~V} 1=2$, consulta: diciembre de 2010]

INSTITUTO NACIONAL DE ESTADÍSTICA (1976 y 2009). Estadisticas sobre la Educación en España.

- (2005). Encuesta de población activa. Base de microdatos disponible en la web. [http://www.ine.es/jaxi/menu.do?type=pcaxis\&path=\%2Ft13\%2Fp405\&file=in ebase $\& \mathrm{~L}=0$ ]

- (2011). Series retrospectivas 2000-2009. CNO 2011. [http://www.ine.es/jaxi/menu. do?type=pcaxis \&path=/t22/e308_mnu\&file=inebase $\& N=\& L=0$, consulta: mayo de 2011]

Johnson, M.K. (2002). «Social Origins, Adolescent Experiences, and Work Value Trajectories During the Transition to Adulthood». Social Forces, 80 (4), 1307-41.

Kalmijn, M. (1994). "Mother's Occupational Status and Children's Schooling». American Sociological Review, 59 (2), 257-75.

Kerbo, H. (2003). Estratificación social y desigualdad. Madrid: McGraw Hill.

KoHn, M.L. (1969). Class and Conformity. Homewood, IL: Dorsey.

- (1976). «Social Class and Parental Values: Another Confirmation of the Relationship». American Sociological Review, 41 (3), 538-45.

- (1977). Class and Conformity a Study in Values, with a Reassessment. 2. ${ }^{\text {a ed. Chi- }}$ cago: University of Chicago Press.

- (1981). «Personality, Occupation, and Social Stratification: A Frame of Reference». En: Treiman, D.J. y Robinson, R.V. (ed.). Research in Social Stratification and Mobility, vol. 1. Greenwich, CT: JAI Press, 267-97.

Kohn, M.L. y Schoenbach, C. (1993). «Social Stratification, Parents' Values and Children's Values». En: Krebs, D. y Schmidt, P. New Directions in Attitude Measurement. New York: Walter de Gruyter. 118-51.

Kohn, M.L. y Schooler, C. (1982). "Job Conditions and Personality: A Longitudinal Assessment of Their Reciprocal Effects». American Journal of Sociology, 87 (6), 1257-86.

- (1983). Work and Personality: An Inquiry into the Impact of Social Stratification. Norwood, NJ: Ablex.

Kohn, M.L.; Naoi, A.; Schoenbach, C.; Schooler, C. y Slomczynski, K.M. (1990). "Position in the Class Structure and Psychological Functioning in the United States, Japan, and Poland». American Journal of Sociology, 95 (4), 9641008.

Masjuan, J.M. (2005). «Progresos en los aprendizajes, características de los estudios y motivaciones de los estudiantes». Papers: Revista de Sociologia, 76, 97-133.

OrganizATION FOr ECONOMIC COOPERATION AND DEVELOPMENT (2007). Education at a Glance 2007. OECD Indicators. París: OECD Publishing.

- (2010). Education at a Glance. OECD Indicators. París: OECD Publishing.

PABlos, L. de; GIL, M. (2007). «Análisis de los condicionantes económicos del acceso a la educación superior». Presupuesto y Gasto Público, 48: 37-57.

PARSONS, T. (1959). "The School Class as Social system: some of its Functions in American Society». Harvard Educational Review, 29 (4), 297-318.

Pearlin, L.J. y KoHn, M.L. (1966). «Social Class, Occupation, and Parental Values: A Cross-National Study». American Sociological Review, 31 (4), 466-79.

PlANAS, J. y FACHELLI, S. (2010). Les universitats catalanes, factor d'equitat i de mobilitat professional: Una anàlisi sobre les relacions entre l'estatus familiar, el bagatge acadèmic 
i la inserció professional l'any 2008 dels titulats l'any 2004 a les universitats catalanes. Barcelona: AQU.

Planas, J.; Giret, J.-F.; Sala, G. y Vincens, J. (2001). «Skils Market, Dynamics and Regulation». En: DesCy, P.; TeSSARING, M. Training in Europe. Second report on vocational training research in Europe 2000. Background report. Cedefop reference series. Office for Official Publications of the European Comunities (3 volumes), 313-382.

Planas, J.; SÁnchez, A. y Ustrell, M. (GRET-UAB) (2011). «El acceso a la Universidad desde la FP». En: X Encuentro de los Servicios de Información y Orientación Universitarios. Universidad de Oviedo, 5 y 6 de mayo de 2011.

Raffe, D. (2003). "Pathways Linking Education and Work: A Review of Concepts, Research, and Policy Debates». Journal of Youth Studies, 6 (1).

- (en prensa). «Itinerarios que relacionan educación con trabajo: Revisión de conceptos, investigación y debates políticos». Papers: Revista de Sociologia.

RAHONA, M. (2009). "Equality of opportunities in Spanish higher education». Higher Education, 58, 285-306.

Rotger, J.M. (2009). «El sistema universitari davant l'espai de Bolonya». En: SUbIRATS, M. (coord.). Societat catalana 2009. Barcelona: Associació Catalana de Sociologia.

SALA, G. (2007). "Approaches to skills mismatch: a literature review». SKOPE. University of Oxford. Working paper.

SALA, G. (2011). «Approaches to skills mismatch in the labour market: A literature review". Papers: Revista de Sociologia, 96 (4).

Sala, G. y Planas, J. (2009). «Retos teóricos e implicaciones metodológicas del enfoque de competencia laboral». Sociologia del Trabajo, 66, 31-46.

Sala, G.; Planas, J.; Masjuan, J.M. y Rodríguez, P. (2007). «El fenomen de la transició laboral». En: Educació superior i treball a Catalunya: Anàlisi dels factors d'inserció laboral. Barcelona: AQU. Generalitat de Catalunya, 9-37.

Sala, G.; Planas, J. y VAN Rompaey, E. (2010). «Especialidad de formación, especialidad de empleo y resultados de inserción. ¿Trabajan los jóvenes donde "deberían"?». Sociología del Trabajo, 70, 87-10.

SAlido CORTÉs, O. (2001). La movilidad ocupacional de las mujeres en España: Por una sociología de la movilidad femenina. Madrid: Centro de Investigaciones Sociológicas.

SlOMCZYNSKI, K.M.; Miller, J. y KOHN, M.L. (1981). «Stratification, Work and Values: A Polish-United States Comparison». American Sociological Review, 46, 720-44.

Subirats, M. (2009). Els nivells educatius de la població i la transmissió del capital cultural. Enquesta Metropolitana de Barcelona 2006. Barcelona: IERM.

WARREN, J. y HAUSER, R. (1997). «Social stratification across three generations. New evidences' from the Wisconsin Longitudinal Study». American Sociological Association, 62, 4.

XIaO, H. (2000). «Class, Gender, and Parental Values in the 1990s». Gender \& Society, 14(6), 785-803. 


\section{Anexo 1. Ficha técnica del estudio ${ }^{6}$}

Los estudios de inserción laboral sobre las universidades catalanas los realiza la Agencia para la Calidad del Sistema Universitario de Catalunya (AQU), sobre la base de encuestas llevadas a cabo desde 2001. La última encuesta fue realizada entre el 16 de enero y el 13 de marzo de 2008 (AQU, 2008: 5). La población encuestada de titulados en el año académico 2003-2004 es de 12.258, aunque, en el caso de la carrera de Medicina, la población de referencia es la promoción 2001, dado que presenta una transición laboral más larga que el resto de los estudios.

- Empresa que ha llevado a término el trabajo de campo: DYM, Market Research.

- Población: graduados de la promoción de 2004 (curso 2003-2004). En el caso de Medicina, por la trayectoria más larga de inserción que presentan, el año de graduación fue el del curso 2000-2001.

- Muestra: se calculó la muestra necesaria para lograr un error muestral por titulación y universidad no superior al $8 \%$. En la práctica, este criterio implica llamar a toda la población de las titulaciones pequeñas (menos de cuarenta titulados) y, para las titulaciones restantes, finalizar las entrevistas una vez lograda la muestra fijada.

- Período: el estudio se ha llevado a cabo entre el 16 de enero y el 13 de marzo de 2008.

- Horario de las llamadas: de 10 a 21 h, más una hora los sábados por la mañana.

- Back office: se han revisado todos los códigos de rama de actividad económica a partir de la codificación abierta.

- Tiempo medio: el tiempo medio de las llamadas fue de quince minutos en los graduados en situación de trabajo; de dieciséis si en el momento de hacer la entrevista no trabajaban pero lo habían hecho tras finalizar los estudios, y de seis minutos si no habían trabajado después de graduarse.

- Tipología de llamadas: en total, se han hecho 132.519 llamadas, con una media de 4 llamadas por entrevista realizada. El 55\% de las entrevistas se ha hecho a teléfonos fijos (6.692) y el 45\%, a teléfonos móviles (5.465). Se han realizado 101 entrevistas en el extranjero.

6. Sobre la base de AQU (2008), Tercer estudi d'inserció laboral dels graduats de les universitats catalanes: Primera valoració dels resultats, Barcelona, Agència per a la Qualitat del Sistema Universitari de Catalunya. 
Tabla 1. Población y muestra por universidad

\begin{tabular}{|c|c|c|c|c|}
\hline Universidad & Población & Muestra & $\begin{array}{l}\text { Respuesta } \\
\text { sobre la } \\
\text { población } \\
(\%)\end{array}$ & $\begin{array}{l}\text { Error } \\
\text { muestral }\end{array}$ \\
\hline Universidad de Barcelona & 7.363 & 3.279 & $44,53 \%$ & $1,27 \%$ \\
\hline Universidad Autónoma de Barcelona & 4.759 & 2.605 & $54,74 \%$ & $1,29 \%$ \\
\hline Universidad Politécnica de Cataluña & 3.594 & 1.694 & $47,13 \%$ & $1,73 \%$ \\
\hline Universidad Pompeu Fabra & 1.682 & 892 & $53,03 \%$ & $2,25 \%$ \\
\hline Universidad de Girona & 1.599 & 1.100 & $68,79 \%$ & $1,65 \%$ \\
\hline Universidad de Lleida & 1.411 & 975 & $69,10 \%$ & $1,74 \%$ \\
\hline Universidad Rovira y Virgili & 1.935 & 1.226 & $63,36 \%$ & $1,69 \%$ \\
\hline Universidad de Vic & 680 & 487 & $71,62 \%$ & $2,37 \%$ \\
\hline Total & 23.023 & 12.258 & $53,24 \%$ & $0,61 \%$ \\
\hline
\end{tabular}

Fuente: AQU (2008: 5). 